\title{
Intraoperative angiography via the popliteal artery: a useful technique for patients in the prone position
}

\author{
Nicolas W. Villelli, MD, ${ }^{1}$ David M. Lewis, MD, ${ }^{1}$ Thomas J. Leipzig, MD, ${ }^{1,2}$ Andrew J. DeNardo, MD, ${ }^{2}$ \\ Troy D. Payner, MD, ${ }^{1,2}$ and Charles G. Kulwin, MD ${ }^{1,2}$ \\ 1Department of Neurological Surgery, Indiana University School of Medicine, Indianapolis; and ${ }^{2}$ Goodman Campbell Brain and \\ Spine, Indianapolis, Indiana
}

\begin{abstract}
OBJECTIVE Intraoperative angiography can be a valuable tool in the surgical management of vascular disorders in the CNS. This is typically accomplished via femoral artery puncture; however, this can be technically difficult in patients in the prone position. The authors describe the feasibility of intraoperative angiography via the popliteal artery in the prone patient.

METHODS Three patients underwent intraoperative spinal angiography in the prone position via vascular access through the popliteal artery. Standard angiography techniques were used, along with ultrasound and a micropuncture needle for initial vascular access. Two patients underwent intraoperative angiography to confirm the obliteration of dural arteriovenous fistulas. The third patient required unexpected intraoperative angiography when a tumor was concerning for a vascular malformation in the cervical spine.

RESULTS All 3 patients tolerated the procedure without complication. The popliteal artery was easily accessed without any adaptation to typical patient positioning for these prone-position cases. This proved particularly beneficial when angiography was not part of the preoperative plan.

CONCLUSIONS Intraoperative angiography via the popliteal artery is feasible and well tolerated. It presents significant benefit when obtaining imaging studies in patients in a prone position, with the added benefit of easy access, familiar anatomy, and low concern for catheter thrombosis or kinking.

https://thejns.org/doi/abs/10.3171/2018.1.SPINE171257
\end{abstract}

KEYWORDS intraoperative angiography; spinal angiography; popliteal artery; diagnostic technique

$\mathrm{D}$ IGITAL subtraction angiography (DSA) is an invaluable tool for the treatment of vascular pathology in the nervous system. Its use before, during, and after surgical intervention provides the opportunity for preoperative planning, as well as confirmation that the appropriate intervention has been completed. Its use is well established in the treatment of intracranial pathologies such as aneurysms ${ }^{1,11}$ and arteriovenous malformations $(\mathrm{AVMs}),{ }^{8,16,17}$ as well as in spinal pathology, most often dural arteriovenous fistulas (dAVFs). ${ }^{7,9}$

The most common position for a patient undergoing DSA is supine, offering easy access to the femoral artery. Intraoperative vascular access in spine surgery creates a challenge because patients are often prone. Although it has been described, femoral access in a patient positioned prone or lateral adds significant challenges to the procedure, including poor sterility at the access site and thrombosis or kinking of the sheath. ${ }^{10}$ Authors have also described obtaining vascular access in the upper extremities through either the brachial or radial artery. ${ }^{10,12}$ This approach, however, does have its limitations, mainly difficulty with navigation across the aortic arch and access to the left common carotid artery when approaching from the left upper extremity.12

We describe 3 cases in which popliteal artery access was used to perform intraoperative spinal angiograms.

ABBREVIATIONS AVM = arteriovenous malformation; dAVF = dural arteriovenous fistula; DSA = digital subtraction angiography; FISH = Femoral Introducer Sheath and Hemostasis; OR = operating room.

SUBMITTED November 17, 2017. ACCEPTED January 30, 2018.

INCLUDE WHEN CITING Published online June 15, 2018; DOI: 10.3171/2018.1.SPINE171257. 
Popliteal canalization has been described for the treatment of peripheral vascular disease, ${ }^{3,5,21}$ but to the best of our knowledge this is the first description of its use for intraoperative angiograms in the treatment of neurovascular pathology. This is a feasible approach that offers easy vascular access to the interventionist and has little associated risk.

\section{Methods}

This case series includes 3 patients who underwent intraoperative spinal angiography via popliteal artery puncture. In the first 2 patients, standard techniques were used to perform the angiogram, with the exception of point of puncture. In the case of the first patient, her popliteal fossa was prepared and draped in a sterile fashion using standard draping. A sterile hemostat was clipped to the edge of the drape lying over the fossa. The area in which the spinal surgery would take place was then prepared and draped in sterile fashion. The spinal draping was placed over the popliteal drape. When the time came for the angiogram, the popliteal fossa was easily found by palpation of the hemostat. The spinal draping over the hemostat was cut, exposing the still-sterile angiogram drape. In the case of the third patient, in whom an intraoperative angiogram had not been planned, the spinal draping was then cut to expose the popliteal fossa. This area was then prepared and draped with standard angiogram draping. A C-arm apparatus was then brought into the operating room (OR) and draped for all cases. The popliteal artery was accessed with a micropuncture kit, then a 5-Fr sheath was introduced, through which diagnostic catheters were advanced to the area of interest by using standard guidewire techniques. Ultrasound was used to access the artery with the micropuncture needle in all cases. Standard anteroposterior and lateral angiograms were then completed. After completion of the angiogram, the microcatheter and sheath were withdrawn and hemostasis was obtained by applying direct pressure to the puncture site. In 1 case, a Femoral Introducer Sheath and Hemostasis (FISH; Morris Innovative) device was used. The remainder of each spinal surgery was then completed in standard form.

\section{Illustrative Cases \\ Case 1}

The first patient was an 81-year-old woman who presented with slowly progressive gait and bladder dysfunction. This eventually led to imaging of the thoracic spine, which demonstrated the presence of tortuous veins and $\mathrm{T} 2$ prolongation (Fig. 1A and B). A subsequent spinal angiogram revealed an AVF at the level of L4 on the right (Fig. 1C). Due to the patient's clinical and radiological findings, it was decided to pursue surgical obliteration of the fistula. The patient was then taken to the OR and placed in a prone position. After the draping technique described above was completed, a midline incision was made overlying the L3 and L4 lamina, and an L3-4 laminectomy was performed. The dura mater was then opened and an arterialized vein was appreciated entering the thecal sac at the level of the right L4 foramen. A temporary clip was applied to the ves-
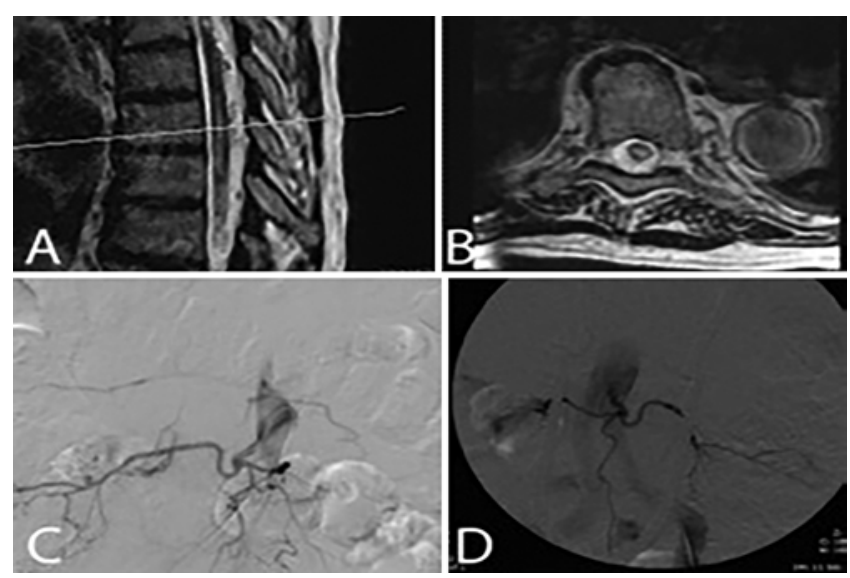

FIG. 1. Sagittal and axial T2-weighted MR images demonstrate tortuous veins within the thecal sac and increased signal in the thoracic spinal cord (A and B). Preoperative diagnostic angiogram shows the presence of a dAVF originating at the level of the right $L 4$ foramen (C). Intraoperative angiogram showing interruption of the fistula with the temporary clip (D).

sel and an intraoperative angiogram was obtained using our previously described popliteal technique (Fig. 1D). This confirmed closure of the fistulous connection. The vessel was then coagulated and cut and the wound was closed using standard techniques. The patient recovered with no new deficits and no evidence of complication from the popliteal puncture. At 8 years postprocedure, the patient has shown no chronic complications for her popliteal access.

\section{Case 2}

The second patient was a 48-year-old woman who presented with progressive right leg weakness and gait difficulties. MRI of her spine showed marked dilation of her dorsal venous system, a concern for a dAVF. This concern prompted a spinal angiogram, which confirmed the presence of the fistula at T5 (Fig. 2A). Because of these symptoms and angiogram findings, the patient was taken to the OR for surgical obliteration of the fistula. The patient was positioned prone and the popliteal fossa was prepared and draped as described above. The patient's thoracic spine was then prepared and draped, and a midline incision was made, followed by a T5-6 laminoplasty. The dura was opened in a normal fashion and a few small arteries were identified around the T5 nerve root. These were transected after bipolar cautery. At this point, it was thought that the fistula had been obliterated, so an intraoperative angiogram was performed through the popliteal artery as described above. Unfortunately, the angiogram showed that the fistula was still patent (Fig. 2B), so further dissection was performed anteriorly. This revealed another artery. A clip was placed on this vessel, which did not affect somatosensory evoked potentials and motor evoked potentials monitoring. Because monitoring remained stable, this vessel was cauterized and cut. A repeat angiogram confirmed obliteration of the fistula (Fig. 2C). The incision was closed in a standard fashion. The patient tolerated the surgery well and was neurologically intact 


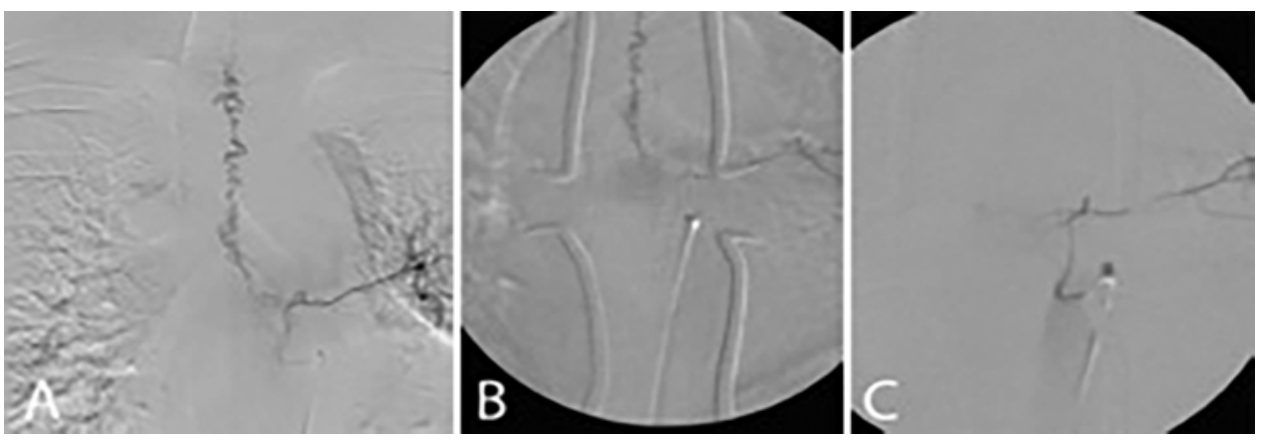

FIG. 2. Preoperative angiogram showing a dAVF originating at the T5 level (A). Intraoperative angiogram showing residual fistula (B). Repeat intraoperative angiogram showing obliteration of the fistula (C).

postoperatively. She showed no complications from the popliteal angiogram. At 3 years postprocedure, she has shown no long-term effects from her popliteal access.

\section{Case 3}

The third patient was a 34-year-old woman who presented with slowly progressive weakness involving all of her extremities over the course of 18 months. The patient also reported paresthesias in all 4 extremities that had begun 4 years prior to presentation. Due to a difficult social situation, she had limited access to health care and had not undergone a thorough workup. Her symptoms continued to progress to the point where they were causing significant difficulty with functioning independently. On examination, the patient's strength was antigravity in her left upper and lower extremities and she could weakly resist in her right upper and lower extremities. An MRI study demonstrated a syringomyelia in the cervical spinal cord, with a weakly enhancing lesion behind C4 (Fig. 3A-D). This was thought to represent an intramedullary neoplasm and plans were made to take the patient to the OR for tumor resection with the use of motor and somatosensory evoked potentials. She was placed prone with her head in a Mayfield apparatus. The midcervical spine was exposed and laminoplasty was performed from $\mathrm{C} 3$ to $\mathrm{C} 5$. Upon opening the dura, there was a large, tortuous vein that had not been visualized on preoperative imaging. It seemed to be originating in the vicinity of the fifth cervical root on the left side, so there was concern that it might be an AVF. In this case, preoperative suspicion for vascular pathology was low, so preparations for an intraoperative angiogram had not been made. Because angiography was necessary to rule out vascular pathology, the draping over the popliteal fossa was cut and the fossa was prepared and draped with angiogram draping. Using the popliteal access technique described above, a vertebral artery angiogram confirmed the absence of any vascular pathology (Fig. 3E). The procedure then proceeded as planned with a midline myelotomy and biopsy. Frozen section suggested a diagnosis of pilocytic astrocytoma, and a subtotal resection
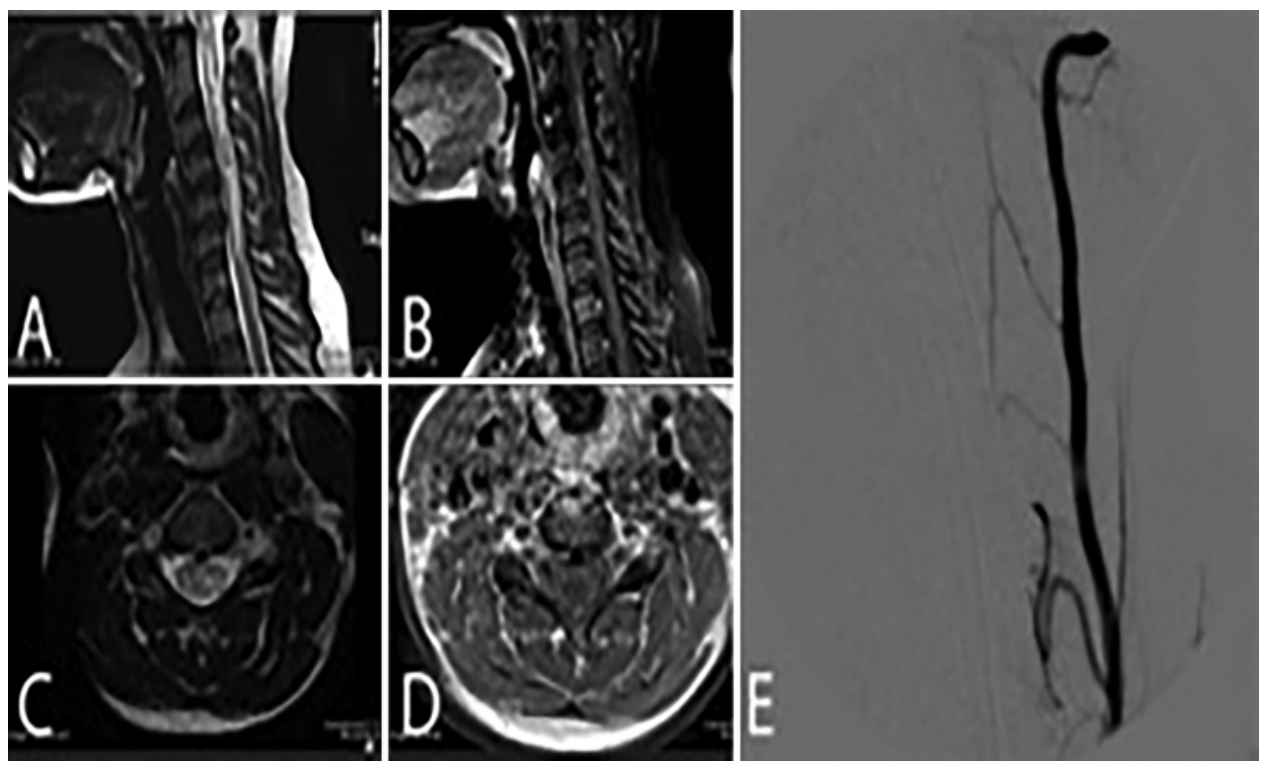

FIG. 3. Sagittal and axial T2-weighted as well as sagittal and axial T1-weighted postcontrast MRI of the cervical spine demonstrating syringomyelia and a faintly enhancing mass lesion at the level of C4 (A-D). Intraoperative angiogram showing normal vasculature of the cervical spine and no sign of $d A V F(E)$. 
was performed due to a lack of a clear plane between the lesion and the spinal cord. We were never able to obtain somatosensory potentials, and motor evoked potentials actually improved during the case. The patient continued to improve throughout her hospitalization and was able to be discharged to an acute rehabilitation facility. She did not have any evidence of complication from the angiogram after the procedure. Long-term complications could not be ruled out because this patient was lost to follow-up.

\section{Discussion}

DSA is the gold-standard procedure to confirm the complete obliteration of neurovascular pathologies such as aneurysms, AVMs, and dAVFs. ${ }^{2,4,8,22}$ Often, it is most helpful to perform the angiogram intraoperatively, to confirm elimination prior to the completion of the surgery. In cases in which the patient is positioned supine, femoral artery access is easily obtained. Various methods for obtaining intraoperative angiograms in lateral or prone positions have been described in neurosurgical patients..$^{10,12}$ Lang et al. compared femoral access to the transradial approach in three-quarters lateral and prone patients..$^{10}$ These investigators showed that both approaches were acceptable, but each had its advantages and disadvantages. The primary advantage to the transfemoral technique was familiarity with the anatomy. The main disadvantages included lack of sterility around the access site, possible thrombosis or kinking of the sheath, and skin injury while the patient's body was resting on the catheter during the procedure. The advantage to using radial artery access is its easier accessibility in the prone position, but the main disadvantages include difficulty crossing an elongated aortic arch and accessing the left common carotid artery when approaching from the left. ${ }^{10,12}$ Popliteal artery access, as used in our patients, provides the advantages of both techniques while avoiding their disadvantages. The popliteal access provides comfort to the interventionist because the artery is readily accessible with the patient in the prone position. It also offers familiar anatomy; the catheter is passed into the femoral artery and advanced similarly to a transfemoral approach, with simple access to all carotid and vertebral arteries.

Intraoperative DSA for spinal vascular lesions is well described. . $^{2,13,15}$ Orru' et al. described a technique of obtaining femoral access while the patient was in the supine position. ${ }^{13}$ Once the sheath was secured to the lateral thigh, the patient was then flipped prone for the spine surgery. The catheter was then accessed on the lateral thigh when intraoperative angiography was needed. Although this is feasible, issues with sheath thrombosis or kinking can arise. ${ }^{10}$ We found intraoperative spinal angiography helpful to confirm lesion obliteration and to rule out the presence of a vascular lesion. Additionally, we did not have to struggle with access or catheter kinking in our popliteal access.

There are two potential intra-angiography issues with this approach, the first of which is the length of catheter needed for the procedure. In high thoracic or vertebral injections, patients may require a longer catheter, but in our case series a catheter longer than the standard $100 \mathrm{~cm}$ was never needed. The second potential issue is the closure. Manual compression may be difficult within the popliteal fossa, but we used this method without issue. In 1 patient a FISH closure system was used. The benefit of this system is that it does not require angiographic evaluation of the vessel, so placement in the OR is feasible. None of our patients experienced any postprocedure hematomas, independent of whether or not a closure device was used. Other closure devices may also work with this approach, but we do not have experience using them in popliteal access. Overall, this approach is not technically difficult and any trained interventionist should be able to perform this access with ease.

Popliteal access has been used in peripheral vascular interventions. ${ }^{3,5,20,21}$ It was first described by Tønnesen et al. in $1988 .^{18}$ This was initially performed for access to the superficial femoral artery and iliac lesions. At 3-year follow-up, there were no differences in complications when compared to other techniques. It has been established as safe for both antegrade and retrograde angiography. ${ }_{6}^{6,23}$ Although studies show that complications are comparable to those in other access areas, it still does have associated risk. Trigaux et al. used cadaveric and CT analysis of the popliteal anatomy to better understand risks associated with a puncture in the popliteal fossa. ${ }^{19}$ They found that the popliteal artery and vein are contained within a common sheath and that the artery lies anteromedial to the vein in $43 \%$ of cases, anterior in $40 \%$ of cases, and anterolateral in $9 \%$ of cases. There is concern that with the proximity of the artery to the vein combined with the difficulty of compression in the popliteal fossa, an AVF could develop after the catheter is removed..$^{14}$ Another potential risk of this access technique is critical limb ischemia. Peripheral pulses and lower-extremity strength examinations should be monitored closely after the procedure to avoid this potential complication. Surgeons consistently recommend the use of a micropuncture needle and ultrasound to obtain access to the popliteal artery. ${ }^{14,20}$ We found this technique to be helpful with our patients and did not experience any major complications in the procedures.

In our patients, popliteal access was used for intraoperative spinal angiograms, but it can also be used for intracranial surgeries when patient positioning is lateral or prone. Certain cranial or craniocervical surgeries require the lateral or prone position. In these surgeries, a nonfemoral vascular access point may be more suitable. Nossek et al. described the use of radial access for a cerebellar AVM and tentorial dAVF. ${ }^{12}$ In these cases, the authors found radial artery access easily obtained, but did encounter issues with navigation across an elongated aortic arch. Our popliteal approach offers easy access but avoids the issue of navigating a difficult aorta in both cranial and spine intraoperative angiograms.

\section{Conclusions}

The popliteal artery provides easy access for intraoperative angiograms in patients positioned lateral or prone, and it also provides familiar anatomy and low risk of complications, both periprocedural and long-term. Its use allows for simple confirmation of vascular obliteration in neurovascular surgeries of both the brain and spine. 


\section{References}

1. Alexander TD, Macdonald RL, Weir B, Kowalczuk A: Intraoperative angiography in cerebral aneurysm surgery: a prospective study of 100 craniotomies. Neurosurgery 39:10-18, 1996

2. Barrow DL, Boyer KL, Joseph GJ: Intraoperative angiography in the management of neurovascular disorders. Neurosurgery 30:153-159, 1992

3. Brountzos EN, Moulakakis KG, Avgerinos ED, Dalainas I, G Giannakopoulos T, Kakisis J, et al: Retrograde transpopliteal approach of iliofemoral lesions. Vasc Endovascular Surg 45:646-650, 2011

4. Chalouhi N, Theofanis T, Jabbour P, Dumont AS, Fernando Gonzalez L, Starke RM, et al: Safety and efficacy of intraoperative angiography in craniotomies for cerebral aneurysms and arteriovenous malformations: a review of 1093 consecutive cases. Neurosurgery 71:1162-1169, 2012

5. Fanelli F, Lucatelli P, Allegritti M, Corona M, Rossi P, Passariello R: Retrograde popliteal access in the supine patient for recanalization of the superficial femoral artery: initial results. J Endovasc Ther 18:503-509, 2011

6. Feiring AJ, Wesolowski AA: Antegrade popliteal artery approach for the treatment of critical limb ischemia in patients with occluded superficial femoral arteries. Catheter Cardiovasc Interv 69:665-670, 2007

7. Fox S, Hnenny L, Ahmed U, Meguro K, Kelly ME: Spinal dural arteriovenous fistula: a case series and review of imaging findings. Spinal Cord Ser Cases 3:17024, 2017

8. Hashimoto H, lida J, Hironaka Y, Sakaki T: Surgical management of cerebral arteriovenous malformations with intraoperative digital subtraction angiography. J Clin Neurosci 7 (Suppl 1):33-35, 2000

9. Koch MJ, Stapleton CJ, Agarwalla PK, Torok C, Shin JH, Coumans JV, et al: Open and endovascular treatment of spinal dural arteriovenous fistulas: a 10 -year experience. J Neurosurg Spine 26:519-523, 2017

10. Lang SS, Eskioglu E, Mericle RA: Intraoperative angiography for neurovascular disease in the prone or three-quarter prone position. Surg Neurol 65:283-289, 2006

11. Macdonald RL, Wallace MC, Kestle JR: Role of angiography following aneurysm surgery. J Neurosurg 79:826-832, 1993

12. Nossek E, Chalif DJ, Buciuc R, Gandras EJ, Anderer EG, Insigna $S$, et al: Intraoperative angiography for arteriovenous malformation resection in the prone and lateral positions, using upper extremity arterial access. Oper Neurosurg (Hagerstown) 13:352-360, 2017

13. Orru' E, Sorte DE, Wolinsky JP, Jallo GI, Bydon A, Tamargo RJ, et al: Intraoperative spinal digital subtraction angiography: indications, technique, safety, and clinical impact. J Neurointerv Surg 9:601-607, 2017

14. Saha S, Gibson M, Magee TR, Galland RB, Torrie EP: Early results of retrograde transpopliteal angioplasty of iliofemoral lesions. Cardiovasc Intervent Radiol 24:378-382, 2001

15. Schievink WI, Vishteh AG, McDougall CG, Spetzler RF: Intraoperative spinal angiography. J Neurosurg 90 (1 Suppl):48-51, 1999

16. Starke RM, Komotar RJ, Hwang BY, Fischer LE, Garrett MC, Otten ML, et al: Treatment guidelines for cerebral ar- teriovenous malformation microsurgery. Br J Neurosurg 23:376-386, 2009

17. Suzuki H, Maki H, Taki W: Evaluation of cerebral arteriovenous malformations using image fusion combining threedimensional digital subtraction angiography with magnetic resonance imaging. Turk Neurosurg 22:341-345, 2012

18. Tønnesen KH, Sager P, Karle A, Henriksen L, Jørgensen B: Percutaneous transluminal angioplasty of the superficial femoral artery by retrograde catheterization via the popliteal artery. Cardiovasc Intervent Radiol 11:127-131, 1988

19. Trigaux JP, Van Beers B, De Wispelaere JF: Anatomic relationship between the popliteal artery and vein: a guide to accurate angiographic puncture. AJR Am J Roentgenol 157:1259-1262, 1991

20. Vatakencherry G, Gandhi R, Molloy C: Endovascular access for challenging anatomies in peripheral vascular interventions. Tech Vasc Interv Radiol 19:113-122, 2016

21. Wijeyaratne SM, Wijewardene N: Endovascular stenting of a persistent sciatic artery aneurysm via retrograde popliteal approach: a durable option. Eur J Vasc Endovasc Surg 38:91-92, 2009

22. Yanaka K, Matsumaru Y, Okazaki M, Noguchi S, Asakawa $\mathrm{H}$, Anno I, et al: Intraoperative angiography in the surgical treatment of cerebral arteriovenous malformations and fistulas. Acta Neurochir (Wien) 145:377-383, 2003

23. Younes HK, El-Sayed HF, Davies MG: Retrograde transpopliteal access is safe and effective-it should be added to the vascular surgeon's portfolio. Ann Vasc Surg 29:260-265, 2015

\section{Disclosures}

The authors report no conflict of interest concerning the materials or methods used in this study or the findings specified in this paper.

\section{Author Contributions}

Conception and design: Villelli, Leipzig, DeNardo, Payner, Kulwin. Acquisition of data: Villelli, Lewis. Analysis and interpretation of data: Villelli, Kulwin. Drafting the article: Villelli. Critically revising the article: Villelli, Kulwin. Reviewed submitted version of manuscript: all authors. Approved the final version of the manuscript on behalf of all authors: Villelli. Statistical analysis: Villelli. Administrative/technical/material support: all authors. Study supervision: Villelli, Leipzig, DeNardo, Payner, Kulwin.

\section{Supplemental Information Previous Presentations}

Portions of this work were presented as an electronic poster at the Annual Scientific Meeting of the American Association of Neurological Surgeons in April 2014 in San Francisco, California.

\section{Correspondence}

Nicolas W. Villelli: Indiana University School of Medicine, Indianapolis, IN.nwvillel@iupui.edu. 